\title{
UNIFORM APPROXIMATION IN VARIOUS FUNCTION CLASSES
}

\author{
OLAV NJASTAD
}

J. E. Fenstad (in [5], [6], [7]) has investigated the structure of the system of all uniformly continuous real-valued functions on a uniform space. These systems are 1 -groups, a fact which makes available a well developed general representation theory. As is pointed out in [7], the system of all real-valued $p$-continuous functions on a proximity space need not be a group or a lattice. The task of giving an algebraic characterization of these systems thus appears considerably more difficult. The same remarks apply to the systems of all real-valued uniformly continuous mappings of generalized uniform spaces, which were introduced in [2] in order to simplify the connection between uniform structures and proximity structures and to obtain a unified theory of completion. This paper is primarily concerned with establishing uniform approximation theorems in these systems (Propositions 2 and 3, Theorem 1). These may be specialized to (proper) uniform spaces (Theorem 2 , cf. also Theorem 4,1 of [5]), to proximity spaces (Theorem 3), and to completely regular topological spaces (Theorems 4-5, cf. also Theorems 3,5 of [3]).

The development is in terms of uniform coverings. The setting thus differs from that of J. E. Fenstad both in primary object of investigation and in means of exposition, but many of the ideas have their origin in his papers.

1.

We start by recording some fundamental concepts from the theory of coverings of a set $X$ (cf. [9], [10], [11]). The covering $u$ is a refinement of the covering $v$ - written $u<v$ - if each element of $u$ is contained in some element of $v$. If $u$ is a covering, and $A$ a subset of $X$, then the star $\operatorname{St}(A, u)$ of $A$ with respect to $u$ is defined by the formula

$$
\operatorname{St}(A, u)=\bigcup\{U \in u: U \cap A \neq \emptyset\} .
$$

The covering $\{\operatorname{St}(U, u): U \in u\}$ is denoted $u^{*}$. If $u^{*}$ is a refinement of $v$, 
then $u$ is called a star-refinement of $v$-written $u<* v$. A covering - or more generally: a family of sets - $u$ is called star-finite if every $U$ in $u$ meets only a finite number of sets in $u$.

The product of two coverings $u$ and $v$ is defined as

$$
u \wedge v=\{U \cap V: U \in u, V \in v\} .
$$

A generalized uniformity on $X$ may be defined as a collection $\mathscr{U}$ of coverings which satisfies the following conditions:

G 1. If $u \in \mathscr{U}, u<v$, then $v \in \mathscr{U}$.

G 2. If $u \in \mathscr{U}$, there exists $a v \in \mathscr{U}$ such that $v<* u$.

G 3. If $u$ and $v$ are finite coverings in $\mathscr{U}$, then $u \wedge v \in \mathscr{U}$. (Cf. [2, p. 251].)

The coverings in $\mathscr{U}$ are said to be uniform (with respect to $\mathscr{U}$ ). A collection $\mathscr{V}$ satisfying $G 2$ and $G 3$ forms a base for the generalized uniformity obtained by adding all coverings which possess refinements in $\mathscr{V}$.

A generalized uniform space $(X, \mathscr{U})$ is a set $X$ equipped with a generalized uniformity $\mathscr{U}$. (The fundamental properties of generalized uniform spaces are developed in [2], there formulated in terms of entourages).

We recall that a (proper) uniformity is a collection $\mathscr{U}$ of coverings satisfying $G 1, G 2$ and

G $3^{\prime}$. If $u$ and $v$ are arbitrary coverings in $\mathscr{U}$, then $u \wedge v \in \mathscr{U}$.

A sequence $\left\{u_{n}: n=1,2, \ldots\right\}$ of coverings is called normal if $u_{n+1}<* u_{n}$ for all $n$. Evidently a normal sequence is a base for a uniformity. If $u$ is a covering and $\left\{u_{n}: n=1,2, \ldots\right\}$ is a normal sequence such that $u_{1}<* u$, then this sequence - as well as the uniformity for which it is a base - will be said to be associated with the covering $u$. By G 2, every covering $u$ of a generalized uniformity $\mathscr{U}$ admits an associated normal sequence contained in $\mathscr{U}$ (otherwise stated, it admits an associated uniformity coarser than $\mathscr{U}$ ).

If $f$ is a mapping of a set $X$ into a set $Y$, then the inverse image $f^{-1}(v)$ of a covering $v$ of $Y$ is defined to be the covering

$$
f^{-1}(v)=\left\{f^{-1}(V): V \in v\right\} .
$$

A mapping $f$ of a generalized uniform space $(X, \mathscr{U})$ into a generalized uniform space $(Y, \mathscr{V})$ is uniformly continuous if $\mathscr{U}$ contains the inverse image of every covering in $\mathscr{V}$. A family $\left\{f_{j}: j \in I\right\}$ is uniformly equicontinuous - or briefly uniform - if for every $v$ in $\mathscr{V}$ there exists a $u$ in $\mathscr{U}$ which refines all $f_{j}^{-1}(v), j \in I$. A finite family of uniformly continu- 
ous mappings is not uniform in general (see section 5), although this is the case if $\mathscr{U}$ is a proper uniformity.

\section{2.}

Every pseudo-metric $d$ on $X$ determines a uniformity $\mathscr{U}_{d}$ having as a base all coverings $d_{\varepsilon}, \varepsilon>0$, where $d_{\varepsilon}$ consists of all $\varepsilon$-neighbourhoods of points in $X$. In particular, the ordinary metric uniformity on the set $R$ of real numbers has a base consisting of all coverings of the form

$$
v_{\varepsilon}=\{\langle x-\varepsilon, x+\varepsilon\rangle: x \in R\} .
$$

We shall use the letter $R$ to denote the set of real numbers as well as this set equipped with the uniformity described.

The set of all uniformly continuous functions of a generalized uniform space $(X, \mathscr{U})$ into $R$ will be denoted by $U(X, \mathscr{U})$. All the sets of real-valued functions considered, are thought of as subsystems of $R^{X}$, with respect to the (point-wise) lattice- and vector space operations, as far as they are defined. The constant mapping with value $\varrho$ will simply be denoted $\varrho$.

The system $U(X, \mathscr{U})$ needs not be a group or a lattice. (For an example, see section 5). Such anomalies are, of course, only possible when $\mathscr{U}$ is not a proper uniformity. In any case, $U(X, \mathscr{U})$ contains all constant functions and is closed under formation of absolute values and under multiplication with real numbers. Subsets of $R^{X}$ with these three properties will be called $m$-systems. In order to state some additional properties of $U(X, \mathscr{U})$, we give the following definitions.

A real-valued function $f$ is said to separate the sets $A$ and $B$ if

$$
0 \leqq f \leqq 1, \quad f(A)=\{1\}, \quad f(B)=\{0\},
$$

or vice versa. The family $\left\{f_{U}: U \in u\right\}$ is said to separate the covering $u$ if

$$
0 \leqq f_{U} \leqq 1, \quad f_{U}(U)=\{1\}, \quad f_{U}(X-\operatorname{St}(U, u))=\{0\}
$$

for every $U$ in $u$.

Let $u$ be a covering in the generalized uniformity $\mathscr{U}$, and let $\left\{f_{j}: j \in I\right\}$ be a family of real-valued functions on $X$. We shall say that the family is $u$-uniform if it is uniform with respect to some uniformity $\mathscr{U}_{u}$ contained in $\mathscr{U}$ and associated with $u$.

Let $w_{\varepsilon}$ denote the covering consisting of all intersections of sets in the coverings $f_{j}^{-1}\left(v_{\varepsilon}\right)$. Clearly $w_{\varepsilon}$ is refined by all coverings refining all $f_{j}^{-1}\left(v_{\varepsilon}\right)$, and $w_{\varepsilon}$ itself refines all $f_{j}^{-1}\left(v_{\varepsilon}\right)$. Thus if the family $\left\{f_{j}: j \in I\right\}$ is uniform (with respect to $\mathscr{U}$ ), then $w_{\varepsilon} \in \mathscr{U}$. One verifies that $w_{\varepsilon / 2}<{ }^{*} w_{\varepsilon}$. 
Thus $\left\{w_{2^{-n}}: n=1,2, \ldots\right\}$ is a normal sequence associated with $w_{0}$. It follows that $\left\{f_{j}: j \in I\right\}$ is uniform if and only if it is $u$-uniform with respect to some $u$ in $\mathscr{U}$. For a proper uniformity $\mathscr{U}$, this implies that $\left\{f_{j}: j \in I\right\}$ is $u$-uniform for any $u$ in $\mathscr{U}$. In the case of generalized uniformities, it will be of interest to study families which are $u$-uniform for some, but not necessarily all of the coverings $u$ in $\mathscr{U}$ (cf. prop. 2).

We now state a lemma on suprema of families of real numbers.

Lemma 1. Let $\left\{a_{j}: j \in I\right\}$ and $\left\{b_{j}: j \in I\right\}$ be bounded families of real numbers. Then

$$
\left|\sup \left\{a_{j}: j \in I\right\}-\sup \left\{b_{j}: j \in I\right\}\right| \leqq \sup \left\{\left|a_{j}-b_{j}\right|: j \in I\right\} .
$$

The proof is elementary and will be omitted (see e.g. [5, p. 437]).

Proposition 1. The m-system $U(X, \mathscr{U})$ possesses the following properties:

(1) The sum of any finite uniform family in $U(X, \mathscr{U})$ belongs to $U(X, \mathscr{U})$. In particular $f+\varrho \in U(X, \mathscr{U})$ for $\varrho \in R, f \in U(X, \mathscr{U})$.

(2) The supremum of any uniform family in $U(X, \mathscr{U})$ which is pointwise bounded above, belongs to $U(X, \mathscr{U})$. In particular, $\sup (f, \varrho) \in U(X, \mathscr{U})$ for $\varrho \in R, f \in U(X, \mathscr{U})$.

(3) For every uniform covering $u, U(X, \mathscr{U})$ contains a u-uniform family which separates $u$.

Proof. Statement (1) is obvious, while (2) immediately follows from the lemma cited. For the demonstration of (3) we make use of the following fact: Every normal sequence of coverings is determined by a pseudo-metric $d$, in the sense described at the beginning of this section (cf. $\left[4\right.$, p. 15]). Now let $u$ be a uniform covering, $\left\{u_{n}: n=1,2, \ldots\right\}$ a normal sequence associated with it and contained in $\mathscr{U}$, and $d$ a pseudometric determining the uniformity defined by the sequence. There is a $\delta>0$ such that $d_{\delta}<u$. For every $U$ in $u$ we define

$$
f_{U}(x)=\inf \left\{1, \delta^{-1} d(x, X-\operatorname{St}(U, u))\right\} .
$$

Clearly the family $\left\{f_{U}: U \in u\right\}$ separates $u$. Since

$$
|d(x, A)-d(y, A)| \leqq d(x, y),
$$

it easily follows that the family $\left\{f_{u}: U \in u\right\}$ is uniform with respect to $\mathscr{U}_{d}$, hence $u$-uniform.

Remark. An easy argument shows that if the family $\{f, g\}$ is $u$-uniform, so is the family $\{f, g, \sup (f, g), f+g, f-g\}$. Since $\sup (f, g)=$ 
$\frac{1}{2}(f+g)+\frac{1}{2}|f-g|$, we may conclude that if an $m$-system is closed under addition of finite $u$-uniform families, then it is also closed under the formation of suprema of such families.

3.

We now embark on our primary task: the uniform approximations theorems. To this end we need some more concepts.

The cozero-set $C(f)$ of a real-valued function $f$ on $X$ is defined as

$$
C(f)=\{x \in X: f(x) \neq 0\} .
$$

Further, we introduce a rather special kind of uniform coverings on a generalized uniform space $(X, \mathscr{U})$. We remark that the coverings $u_{\varepsilon}$ of $R$, where

$$
u_{\varepsilon}=\{\langle(n-1) \varepsilon,(n+1) \varepsilon\rangle: n=0, \pm 1, \pm 2, \ldots\}, \quad \varepsilon>0,
$$

is a base for the ordinary uniformity on $R$. For every $f \in U(X, \mathscr{U})$ and every $u_{\varepsilon}$ we define

$$
u(f, \varepsilon)=f^{-1}\left(u_{\varepsilon}\right) .
$$

A covering of this type will be called functionally determined. It is not easy to give an intrinsic description of such coverings, and we shall use them only provisionally. The main theorems will be formulated in terms of an important and easily described class of uniform coverings: those which are star-finite and countable. (We remark that the word countable shall always mean "finite or countably infinite".)

Proposition 2. If $S$ is an m-system in $U(X, \mathscr{U})$ such that for every functionally determined covering $u$

(1) $S$ is closed under addition of finite, u-uniform families,

(2) $S$ is closed under formation of suprema of countable, u-uniform families

with the property that the corresponding families of cozero-sets form star-finite coverings of $X$ refined by $u$,

(3) $S$ contains a u-uniform family which separates $u$, then $S$ is uniformly dense in $U(X, \mathscr{U})$.

Proof. Let $f \in U(X, \mathscr{U}), \varepsilon>0$, and set $u=u(f, \varepsilon)$. By condition (3), $S$ contains a $u$-uniform family $\left\{g_{U}: U \in u\right\}$ which separates $u$. We now define

$$
A_{n}=f^{-1}(\langle(n-1) \varepsilon,(n+1) \varepsilon\rangle) .
$$

For $n>0, i \geqq 0$, we define

and for every $n>0$

$$
A_{n}{ }^{i}=\bigcup_{j=-i}^{i} A_{2 n+j}
$$




$$
\mathscr{A}_{n}=\left\{A_{n}{ }^{i}: i=0,1, \ldots, n\right\} .
$$

Now fix an $n>0$, and let $A \in \mathscr{A}_{n}$. We define

$$
f_{A}{ }^{n}=\sup \left\{g_{U}: U \subset A\right\} .
$$

According to (1) and the remark following the proof of Prop. $1, f_{A}{ }^{n} \in S$. We observe that $f_{A}{ }^{n}(x)=1$ for $x \in A, f_{A}{ }^{n}(x)=0$ for $x \notin \operatorname{St}(A, u), 0 \leqq f_{A}{ }^{n} \leqq 1$. Let $\mathscr{U}_{u}$ be a uniformity in $\mathscr{U}$ with respect to which the family $\left\{g_{U}: U \in u\right\}$ is uniformly equicontinuous. It follows from Lemma 1 that the family $\left\{f_{A}{ }^{n}: A \in \mathscr{A}_{n}, n=1,2, \ldots\right\}$ is uniformly equicontinuous with respect to $\mathscr{U}_{u}$.

Now for $n \leqq 0$ we write

and for $n>0$ :

$$
f_{n}=2 \varepsilon g_{A_{n}}
$$

$$
f_{n}=\sum_{A \in \mathscr{A}_{n}} 2 \varepsilon f_{A}^{n}
$$

In this latter case, $f_{n}(x)=2(n+1) \varepsilon$ for $x \in A_{n}{ }^{0}=A_{2 n}, f_{n}(x)=0$ for $x \notin A_{n}{ }^{n+1}$, and $0 \leqq f_{n} \leqq 2(n+1) \varepsilon$.

We observe that if a set in $u$ contains both $x$ and $y$, then $f_{A}{ }^{n}(x) \neq f_{A}{ }^{n}(y)$ for at most two $A \in \mathscr{A}_{n}$. Now for every $\delta>0$ there is a $w \in \mathscr{U}_{u}, w<u$, such that

$$
\left|f_{A}^{n}(x)-f_{A}^{n}(y)\right|<\frac{1}{2} \varepsilon
$$

for $x$ and $y$ in one and the same $W \in w ; A \in \mathscr{A}_{n}, n=1,2, \ldots$ But then $x$ and $y$ are contained in the same set of $u$, so it follows from what was said above, that

$$
\left|f_{n}(x)-f_{n}(y)\right|<\varepsilon, \quad n=1,2, \ldots .
$$

This means that the family $\left\{f_{n}: n=0, \pm 1, \pm 2, \ldots\right\}$ is uniformly equicontinuous with respect to $\mathscr{U}_{u}$.

Since $A_{n}{ }^{n+1} \cap A_{m}{ }^{m+1}=\emptyset$ for $n-2>3 m+2$, whilst $C\left(f_{n}\right) \subset A_{n}{ }^{n+1}$ for $n>0$, and $C\left(f_{n}\right) \subset \operatorname{St}\left(A_{m}, u\right)$ for $n \leqq 0$, the family $\left\{C\left(f_{n}\right): n=0, \pm 1, \pm 2, \ldots\right\}$ is star-finite. Further $f_{n}(x) \neq 0$ for $x \in A_{n}, n \leqq 0$, and $f_{n}(x) \neq 0$ for $x \in A_{n}{ }^{1}$, $n>0$. As

$$
\left\{A_{n}: n=0,-1,-2, \ldots\right\} \cup\left\{A_{n}{ }^{1}: n=1,2, \ldots\right\}
$$

is a covering of $X$, so is $\left\{C\left(f_{n}\right): n=0, \pm 1, \pm 2, \ldots\right\}$. Clearly this covering is refined by $u$, so condition (2) implies that

$$
g=\sup \left\{f_{n}: n=0, \pm 1, \pm 2, \ldots\right\} \in S .
$$

Now let $x \in A_{m}{ }^{1}, m>0$, and $n \geqq m$. One sees that $x \notin A_{n}{ }^{i}$ for $i<2 n-$ $2 m-2$, and so $f_{A}^{n}(x)=0$ for $A=A_{n}{ }^{i}, i=0,1, \ldots, 2 n-2 m-4$. Thus

$$
f_{n}(x) \leqq 2[n+1-(2 n-2 m-4)] \varepsilon \leqq 2(m+5) \varepsilon .
$$


For $n<m$ we have $f_{n}(x)<2(n+1) \varepsilon \leqq 2 m \varepsilon$. As $f_{m}(x) \geqq 2 m \varepsilon$, it follows that $g(x) \in[2 m \varepsilon,(2 m+10) \varepsilon]$, and since $f(x) \in\langle(2 m-2) \varepsilon,(2 m+2) \varepsilon\rangle$, we conclude that $|f(x)-g(x)| \leqq 12 \varepsilon$.

If on the other hand $x \in A_{m}, m \leqq 0$, then we have $g(x) \leqq 2 \varepsilon$. Thus with $f^{+}=\sup \{f, 0\}$ we get $\left|g-f^{+}\right| \leqq 12 \varepsilon$.

An exactly similar construction gives an element $h \in S$ such that $\left|h-f^{-}\right| \leqq 12 \varepsilon$, where $f^{-}=\sup \{-f, 0\}$. Thus

$$
|f-(g-h)| \leqq 24 \varepsilon .
$$

Repeated application of Lemma 1 shows that both $g$ and $h$ are uniformly continuous with respect to the uniformity $\mathscr{U}_{u}$. So $\{g,-h\}$ is a $u$-uniform family, and $g-h$ is in $S$.

It follows that $f \in \bar{S}$, and the proof is completed.

For convenience, we shall adopt the term $l$-space for a lattice-ordered vector space in $R^{X}$ containing all constant function.

Proposition 3. If $S$ is an l-space in $U(X, \mathscr{U})$ such that for every functionally determined covering $u$

(1) $S$ is closed under the formation of suprema of countable families with the property that the corresponding families of cozero-sets form starfinite coverings of $X$ refined by $u$,

(2) $S$ contains a family which separates $u$, then $S$ is uniformly dense in $U(X, \mathscr{U})$.

Proof. Inspection of the proof of Prop. 2 reveals that the operations performed on the initial separating family either are finite or of the type admitted in condition (1). Thus one may start out with an arbitrary family which separates $u$, and construct functions $g, h$ in $S$ such that $|f-(g-h)|<24 \varepsilon$, just as before.

REMark. The closedness conditions imposed on $S$ in prop. 3 are very strong, and the proposition is not very useful in general. It will be used, however, in the special situation of section 6 .

4.

We now state the main theorems. We will call a family $\left\{f_{j}: j \in I\right\}$ star-finite if the family $\left\{C\left(f_{j}\right): j \in I\right\}$ is star-finite, and we will say that it covers $X$ if $\left\{C\left(f_{j}\right): j \in I\right\}$ is a covering of $X$. Since a functionally determined covering is star-finite, immediate specialization of Prop. 2 yields:

Theorem 1. Let $(X, \mathscr{U})$ be a generalized uniform space. If $S$ is an $m$ system in $U(X, \mathscr{U})$ such that for every star-finite, uniform covering $u$ 
(1) $S$ is closed under addition of finite, u-uniform families,

(2) $S$ is closed under formation of suprema of countable, star-finite, u-uniform families which cover $X$,

(3) $S$ contains a u-uniform family which separates $u$, then $S$ is uniformly dense in $U(X, \mathscr{U})$.

For proper uniform spaces we have:

Theorem 2. Let $(X, \mathscr{U})$ be a (proper) uniform space. If $S$ is an 1-space in $U(X, \mathscr{U})$ satisfying:

(1) $S$ is closed under formation of suprema of countable, star-finite uniform families which cover $X$,

(2) For every-countable, star-finite uniform covering $u, S$ contains a uniform family which separates $u$, then $S$ is uniformly dense in $U(X, \mathscr{U})$.

Proof. From Prop. 2 and the reasoning following the definition of $u$-uniform families, the theorem is immediately derived.

Remark. In [5] J. E. Fenstad has introduced conditions $A(1)$ and $A(2)$ which together secure uniform density of an 1 -space $S$ in $U(X, \mathscr{U})$, in the case of a proper uniformity $\mathscr{U}$. (Actually, the slightly more general situation where $S$ is a lattice-ordered group containing all rational constants, is considered.) In order to formulate these conditions, we record some definitions from [5]. A strong u-cover is an ordered pair $(V, u)$, where $u$ is a covering of $X, V$ an entourage of the uniformity and there exists a number $n$ such that for every $A \in u, V(A) \cap B \neq \emptyset$ for at most $n$ sets $B \in u$. The cardinal number $m(X, \mathscr{U})$ is defined as the least infinite cardinal (strictly) greater than the cardinal of any strong $u$-cover. Now the conditions $A(1)$ and $A(2)$ are as follows:

$A(1)$ For each entourage $V$ of the uniformity, and each family $\mathscr{F}$ of subsets, where card $\mathscr{F}<m(X, \mathscr{U})$, there is a uniform family $\left\{f_{A}: A \in \mathscr{F}\right\}$ in $S$ such that $f_{A}$ separates $A$ and $X-V(A)$.

$A(2) S$ is closed under formation of suprema of star-finite (locally finite in the terminology of [5]) uniform families of cardinality strictly less than $m(X, \mathscr{U})$.

By the argument following Theorem 2, these two conditions for $m(X, \mathscr{U})>\boldsymbol{\aleph}_{0}$ imply conditions (2) and (3) of Prop. 2. If on the other hand $m(X, \mathscr{U})=\boldsymbol{\kappa}_{0}$, that is, all strong $u$-covers are finite, we see that all functionally determined coverings are finite. Every star-finite covering with a finite refinement is easily seen to be finite. Thus $A(1)$ and $A(2)$ entail (2) and (3) of Prop. 2 also in this case. So we may conclude: 
If $(X, \mathscr{U})$ is a (proper) uniform space and $S$ an 1-space in $U(X, \mathscr{U})$ which satisfies $A(1)$ and $A(2)$, then $S$ is uniformly dense in $U(X, \mathscr{U})$. (Cf. [5, p. 438].)

5.

We consider a proximity space $(X, \mathscr{P})$. A covering $u$ of $X$ shall be called a proximity covering if

$$
A \Subset \operatorname{St}(A, u) \quad \text { for all } A \subset X .
$$

(It may be verified that the finite proximity coverings coincide with the $p$-coverings of $[1, \mathrm{p} .353]$, or the $\delta$-coverings of $[10$, p. 559].) A proximity covering is normal if it possesses an associated normal sequence of proximity coverings.

If the proximity $\mathscr{P}$ is deduced from the generalized uniformity $\mathscr{U}$ by the formula

$$
A \Subset B(\mathscr{P}) \Leftrightarrow \exists u \in \mathscr{U}: \operatorname{St}(A, u) \subset B,
$$

then $\mathscr{U}$ is said to be compatible with $\mathscr{P}$. The collection of all normal proximity coverings (with respect to a proximity $\mathscr{P}$ ) constitutes a generalized uniformity $\mathscr{U}_{\alpha}$, and $\mathscr{U}_{\alpha}$ is the finest generalized uniformity compatible with the proximity (cf. [2, p. 241]).

A mapping $f$ of a proximity space $(X, \mathscr{P})$ into a proximity space $(Y, \mathscr{Q})$ is $p$-continuous if

$$
A \Subset B(\mathscr{Q}) \Rightarrow f^{-1}(A) \Subset f^{-1}(B)(\mathscr{P}),
$$

(cf. e.g. [1, p. 357]). This is the case if and only if $f$ is uniformly continuous with respect to the corresponding finest generalized uniformities (cf. [2, p. 246]).

The set of all $p$-continuous functions of $(X, \mathscr{P})$ into the ordinary metric proximity space on the set of real numbers will be denoted $P(X, \mathscr{P})$. Now every metric uniformity is the finest generalized uniformity compatible with its proximity ([2, p. 243], [10, p. 570]), so $P(X, \mathscr{P})=U\left(X, \mathscr{U}_{\alpha}\right)$

A family $\left\{f_{j}: j \in I\right\}$ of elements in $P(X, \mathscr{P})$ will be called $u$-uniform if it is uniform with respect to some uniformity which is associated with $u$ and contained in the collection of normal proximity coverings. Because of what was said above, this means exactly that the family is $u$-uniform with respect to the generalized uniformity $\mathscr{U}_{\alpha}$.

From the above remarks and Theorem 1 we obtain:

Theorem 3. Let $(X, \mathscr{P})$ be a proximity space. If $S$ is an m-system in $P(X, \mathscr{P})$ such that for every countable, star-finite, normal proximity covering $u$ 
(1) $S$ is closed under addition of finite, u-uniform families,

(2) $S$ is closed under formation of suprema of countable, star-finite, u-uniform families which cover $X$,

(3) $S$ contains a u-uniform family which separates $u$, then $S$ is uniformly dense in $P(X, \mathscr{P})$.

As J. E. Fenstad has pointed out, the system $P(X, \mathscr{P})$ - and a fortiori $U(X, \mathscr{U})$ - need not be a group or a lattice. For the sake of completeness we sketch a counter-example (cf. [7, p. 135]).

Example. Let $X$ be the set $Z \times Z$, where $Z$ denotes the set of integers. We write $P_{m}=\{m\} \times Z, Q_{n}=Z \times\{n\}$. Let $u$ be a covering consisting of a finite number of unions of sets of the form $P_{m}$, and let $v$ be constructed analoguously from the sets $Q_{n}$. An easy argument shows that the collection of coverings $u \wedge v$, where $u$ and $v$ are of types described, is a base for a generalized uniformity $\mathscr{W}$. Let $\mathscr{P}$ be its associated proximity. We observe that

$$
u_{0}=\left\{P_{m}: m=0, \pm 1, \pm 2, \ldots\right\} \quad \text { and } \quad v_{0}=\left\{Q_{n}: n=0, \pm 1, \pm 2, \ldots\right\}
$$

are normal proximity coverings. Now define

$$
f_{1}(m, n)=m, \quad f_{2}(m, n)=n
$$

and write $g=f_{1}+f_{2}, h=\sup \left\{\left|f_{1}\right|,\left|f_{2}\right|\right\}$. Clearly both $f_{1}$ and $f_{2}$, hence $\left|f_{1}\right|$ and $\left|f_{2}\right|$, are $p$-continuous. We write

$$
D=g^{-1}(\{0\}), \quad E=h^{-1}(\{0, \pm 2, \pm 4, \ldots\}) .
$$

It turns out that $D ほ D(\mathscr{P})$ and $E \xi E(\mathscr{P})$. But

$$
g^{-1}\left(\left\langle-\frac{1}{2}, \frac{1}{2}\right\rangle\right)=D, \quad h^{-1}\left(\bigcup\left\{\left\langle p-\frac{1}{2}, p+\frac{1}{2}\right\rangle: p=0, \pm 2, \pm 4, \ldots\right\}\right)=E,
$$

which shows that neither $g$ nor $h$ are $p$-continuous.

6.

We now consider a completely regular topological space $(X, \mathscr{T})$. An open covering is called normal if it has an associated normal sequence of open coverings. It is well known that the collection of all normal open coverings is a base of a uniformity $\mathscr{U}_{f}$, which is the finest uniformity compatible with the topology. Moreover $U(X, \mathscr{U})=C(X, \mathscr{T})$, where $C(X, \mathscr{T})$ denotes the set of real-valued continuous functions on $(X, \mathscr{T})$.

A family $\left\{f_{j}: j \in I\right\}$ in $C(X, \mathscr{T})$ will be called uniform if it is uniform with respect to some uniformity generated by a normal sequence of open coverings. Because of what was said above, this means exactly that the 
family is uniform with respect to $\mathscr{U}_{f}$. (The concept of $u$-uniformity is not relevant in this connection, since $\mathscr{U}_{f}$ is a proper uniformity).

Application of these remarks to Theorem 2 immediately yields

TheOREM 4. Let $(X, \mathscr{T})$ be a completely regular topological space. If $S$ is an l-space in $C(X, \mathscr{T})$ such that for every countable, star-finite normal open covering $u$

(1) $S$ is closed under formation of suprema of countable, star-finite, uniform families which cover $X$,

(2) $S$ contains a family which separates $u$, then $S$ is uniformly dense in $C(X, \mathscr{T})$.

Two sets $A$ and $B$ are said to be normally separated if there exists a normal open covering $u$ such that $\operatorname{St}(A, u) \cap B=\emptyset$ (or equivalently: $\operatorname{St}(B, u) \cap A=\emptyset)$. This means exactly that $A$ and $B$ are remote in the finest proximity compatible with $\mathscr{T}$, a condition which is equivalent to the following: There exists an $f$ in $C(X, \mathscr{T})$ which separates $A$ and $B$. (The term completely separated is also used for this concept, see e.g. [3, p. 252], $[8$, p. 16].)

Theorem 5. Let $(X, \mathscr{T})$ be a completely regular topological space. If the l-space $S$ in $C(X, \mathscr{T})$ satisfies:

(1) $S$ is closed under formation of suprema of countable star-finite families which cover $X$,

(2) Any two normally separated sets are separated by an element in $S$, then $S$ is uniformly dense in $C(X, \mathscr{T})$.

Proof. Let $u$ be a countable, star-finite, normal, open covering. For every $U$ in $u$, there is an element in $S$ which separates $U$ and $X-\operatorname{St}(U, u)$. That is, $S$ contains a family which separates $u$, and the theorem follows from Prop. 3.

A zero-set is a set of the form $Z=f^{-1}(\{0\})$ for some $f \in C(X, \mathscr{T})$. In terms of zero-sets Theorem 5 admits the following:

Corollary. If the l-space $S$ in $C(X, \mathscr{T})$ satisfies:

(1) $S$ is closed under formation of suprema of countable star-finite families which cover $X$,

(2) Any two disjoint zero-sets are separated by an element in $S$, then $S$ is uniformly dense in $C(X, \mathscr{T})$.

Proof. Any two normally separated sets are contained in disjoint zero-sets (cf. [8, p. 17]). Thus condition (2) of the corollary implies condition (2) of Theorem 5. Condition (1) of the Theorem is explicitly required. 
REMarK. Anderson, in [3], has called a set $S$ which satisfies condition (1) of the corollary $\sigma$-complete, condition (2) normal. Thus the corollary may be formulated: Every normal $\sigma$-complete l-space in $C(X, \mathscr{T})$ is uniformly dense in $C(X, \mathscr{T})$.

Anderson further showed that the normality condition may be weakened to pseudo-normality: For every two disjoint zero-sets $Z_{1}$ and $Z_{2}$, there exists an $f$ in $S$ such that $f^{-1}(\{0\}) \supset Z_{1}, f^{-1}(\langle 0, \infty\rangle) \supset Z_{2}$. (In fact he assumed $S$ to be only a divisible $l$-group.)

We close the section by deducing the vector-lattice version of the ordinary Stone-Weierstrass theorem.

Theorem 6. Let $(X, \mathscr{T})$ be a compact space, and $S$ an l-space in $C(X, \mathscr{T})$. If every two distinct points are separated by an element in $S$, then $S$ is uniformly dense in $C(X, \mathscr{T})$. (Cf. [5, p. 242].)

Proof. In this case every open covering has a finite refinement, hence any star-finite open covering is finite. Thus condition (1) of Theorem 5 is certainly satisfied. If $A$ and $B$ are normally separated, $\bar{A}$ and $\bar{B}$ are disjoint. A standard argument shows that if every two points are separated by an element in an $l$-space $S$ in $C(X, \mathscr{T})$, then also every two disjoint compact sets are separated by an element in $S$ (see e.g. [5, p. 242]). Hence the desired conclusion follows.

7.

Let $(X, \mathscr{U})$ be a generalized uniform space. We proceed to give conditions for a subset $S$ of $U(X, \mathscr{U})$ to contain the whole of $U(X, \mathscr{U})$ (cf. [6]). First we state a lemma on uniformly convergent sequences.

Lemma 2. If $\left\{f_{n}: n=1,2, \ldots\right\}$ is a sequence of bounded elements in $U(X, \mathscr{U})$, which converges uniformly to a bounded element $f \in U(X, \mathscr{U})$, then the family $\left\{f_{n}: n=1,2, \ldots\right\}$ is uniform.

Proof. For every $\varepsilon>0$ we determine an $n_{\varepsilon}$ such that $\left|f_{n}-f\right|<\varepsilon$ for $n>n_{\varepsilon}$. Clearly, we may assume that $n_{\varepsilon_{1}} \geqq n_{\varepsilon_{2}}$ for $\varepsilon_{1}<\varepsilon_{2}$. Now write

$$
w_{\varepsilon}=u(f, \varepsilon) \wedge u\left(f_{1}, \varepsilon\right) \wedge \ldots \wedge u\left(f_{n_{\varepsilon}}, \varepsilon\right) .
$$

Since $f, f_{1}, \ldots f_{n_{\varepsilon}}$ are bounded, we may apply $\mathrm{G} 3$, by which $w_{\varepsilon} \in \mathscr{U}$. If $x$ and $y$ are contained ine one set $\mathrm{W} \in w$, then

for $i \leqq n_{\varepsilon}$, and

$$
\left|f_{i}(x)-f_{i}(y)\right|<2 \varepsilon
$$

$$
\left|f_{i}(x)-f_{i}(y)\right| \leqq\left|f_{i}(x)-f(x)\right|+|f(x)-f(y)|+\left|f(y)-f_{i}(y)\right|<4 \varepsilon
$$


for $i>n_{\varepsilon}$. Since $n_{\frac{1}{2} \varepsilon} \geqq n_{\varepsilon}$, we have $w_{\frac{1}{2 \varepsilon}}<* w_{\varepsilon}$. Thus we conclude that the family $\left\{f_{n}: n=1,2, \ldots\right\}$ is $w_{1}$-uniform, and the proof is completed.

A family $\left\{f_{j}: j \in I\right\}$ is said to be dominated in $S$, if there exists an $f \in S$ such that $\left|f_{j}\right| \leqq f$ for all $j \in I$.

Theorem 7. Let $(X, \mathscr{U})$ be a generalized uniform space, and $S$ an $m$ system in $U(X, \mathscr{U})$. Then $S=U(X, \mathscr{U})$ if and only if:

(1) $S$ is closed under addition of finite uniform families,

(2) Every star-finite, uniform family in $S$ which covers $X$ is dominated in $S$,

(3) $S$ is closed under formation of suprema of uniform families which are dominated in $S$,

(4) For every countable, star-finite, uniform covering u, there is a u-uniform family $\left\{f_{x}: x \in X\right\}$ in $S$ such that $f_{x}(x)=1$ and $f_{x}$ separates $\{x\}$ and $X-\operatorname{St}(\{x\}, u)$.

Proof. The necessity of conditions (1), (2) and (3) immediately follows from Prop. 1, since all the suprema which occur, are finite. Further let $u$ be a countable, star-finite, uniform covering. There exists a starrefinement $v$ of $u$ which is uniform, countable, and star-finite (see [9, p. 153]). Let $\left\{f_{V}: V \in v\right\}$ be a $v$-uniform family in $U(X, \mathscr{U})$ which separates $v$ (such families exist according to Prop. 1). For every $x \in X$, we write $f_{x}=f_{V}$ for some $V$ containing $x$. One easily sees that $f_{x}(x)=1$, that $f_{x}$ separates $\{x\}$ and $X-\operatorname{St}(\{x\}, u)$, and that the family $\left\{f_{x}: x \in X\right\}$ is $u$-uniform. So Prop. 1 also assures the necessity of condition (4).

To prove the sufficiency, let $u$ be a countable, star-finite uniform covering, and let $\left\{f_{x}: x \in X\right\}$ be the uniform family postulated in (4). We define

$$
f_{U}=\sup \left\{f_{x}: x \in U\right\}
$$

for every $U \in u$. All $\left|f_{x}\right| \leqq 1$, so according to (3), $f_{U} \in S$. Clearly $f_{U}$ separates $U$ and $X-\operatorname{St}(U, u)$ and from Lemma 1 follows that the family $\left\{f_{U}: U \in u\right\}$ is $u$-uniform. Thus condition (3) of Theorem 1 is satisfied, while condition (1) is explicitly required, and conditions (3) and (4) of the present theorem imply condition (2). So we conclude that $\bar{S} \supset U(X, \mathscr{U})$.

Next let $f$ be a bounded element in $U(X, \mathscr{U}), f \geqq 0$. There is a sequence $\left\{k_{n}: n=1,2, \ldots\right\}$ in $S$ such that $\left|f-k_{n}\right|<1 /(2 n)$. It follows that

$$
g_{n}=k_{n}-\frac{1}{2 n} \leqq f, \quad\left|f-g_{n}\right| \leqq \frac{1}{n},
$$

and obviously $g_{n} \in S$. We define $f_{n}$ recursively by 


$$
f_{1}=g_{1}, \quad f_{n+1}=\sup \left\{f_{n}, g_{n+1}\right\} \quad \text { for } n>1 .
$$

Assume that $f_{n} \in S$. The coverings

$$
v=f_{n}^{-1}\left(u_{\varepsilon}\right), \quad w=g_{n+1}^{-1}\left(u_{\varepsilon}\right)
$$

are both uniform and finite, hence $v \wedge w \in U$. Since all $\left|g_{n+1}\right| \leqq k_{1}+2$, condition (3) entails that $f_{n+1} \in S$. By induction, we conclude that all $f_{n} \in S$. Now $f=\sup \left\{f_{n}: n=1,2, \ldots\right\}$, hence another application of condition (3) together with Lemma 2 give $f \in S$.

Next let $g \in U(X, \mathscr{U}), g \geqq 0$. It follows from Proposition 1 that $h_{n}=\inf \{g, n\} \in U(X, \mathscr{U})$ for all $n$. As $h_{n}$ is bounded and non-negative, it belongs to $S$. Further there is a $k \in S$ such that $|g-k| \leqq 1$, since $g \in \bar{S}$. Thus $\left|h_{n}\right| \leqq k+1$ for all $n$, and as the family $\left\{h_{n}: n=1,2, \ldots\right\}$ clearly is $u(g, 1)$-uniform, $g=\sup \left\{h_{n}: n=1,2, \ldots\right\} \in S$.

Finally let $h \in U(X, \mathscr{U})$. Then $h^{+}$and $h^{-}$both belong to $S$. The family $\left\{h^{+},-h^{-}\right\}$is $u(h, 1)$-uniform, and so $h^{+}-h^{-} \in S$. This completes the proof.

Naturally, analoguous theorems may be formulated for the other cases treated.

\section{REFERENCES}

1. E. M. Alfsen and J. E. Fenstad, On the equivalence between proximity structures and totally bounded uniform structures, Math. Scand. 7 (1959), 353-360.

2. E. M. Alfsen and O. Njåstad, Proximity and generalized uniformity, Fund. Math. 52 (1963), 235-252.

3. F. W. Anderson, Approximation in systems of real-valued continuous functions, Trans. Amer. Math. Soc. 103 (1962), 249-271.

4. N. Bourbaki, Topologie générale, Ch. IX (Act. Sci. et Ind. 1045) Paris, 1958.

5. J. E. Fenstad, On l-groups of uniformly continuous functions I, Approximation theory, Math. Zeitschr. 82 (1963), 434-444.

6. J. E. Fenstad, On l-groups of uniformly continuous functions II, Representation theory, Math. Zeitschr. 83 (1964), 45-56.

7. J. E. Fenstad, On l-groups of uniformly continuous functions III, Proximity spaces, Math. Zeitschr. 83 (1964), 133-139.

8. L. Gillman and M. Jerison, Rings of continuous functions, New York, 1960.

9. Seymor Ginsburg and J. R. Isbell, Some operators on uniform spaces, Trans. Amer. Math. Soc. 93 (1959), 145-168.

10. Yu. M. Smirnov, On proximity spaces, Math. Sbornik N.S. 31 (1952) 543-574. (Russian.)

11. J. Tukey, Convergence and uniformity in topology, Princeton, 1940. 